\title{
Complex Network of Earthquakes
}

\author{
Sumiyoshi $\mathrm{Abe}^{1}$ and Norikazu Suzuki² \\ 1 Institute of Physics, University of Tsukuba, Ibaraki 305-8571, Japan, \\ suabe@sf6.so-net.ne.jp \\ 2 College of Science and Technology, Nihon University, Chiba 274-8501, Japan \\ suzu@phys.ge.cst.nihon-u.ac.jp
}

\begin{abstract}
The complex evolving network is constructed from the seismic data taken in southern California, and its topological properties are studied. It is shown that the network associated with earthquakes is scale free. The value of the degrees of separation, i.e., the characteristic path length, is found to be small, between 2 and 3. The clustering coefficient is also calculated and is seen to be about 10 times larger than that in the case of the completely random network. These discoveries should play an important role in modeling earthquake phenomenon.
\end{abstract}

\section{Introduction}

Earthquake phenomenon has been attracting much attention from the viewpoint of science of complexity. [1]-[5]. Though seismicity has diverse physical aspects, some known empirical laws are remarkably simple. The Omori law for the temporal pattern of aftershocks [6] and the Gutenberg-Richter law for the relationship between frequency and magnitude [7] are celebrated classical examples. These are the power laws and represent the scale-free natures of seismicity.

In recent papers [8], [9], we have studied spatio-temporal complexity of seismicities in southern California and Japan based on nonextensive statistical mechanics 10]-15, which is considered to be a consistent and unified framework for the statistical description of complex systems. We have found that both the spatial distance [8] and time interval [9] between two successive earthquakes are described extremely well by the so-called $q$-exponential distribution (see below) which maximizes the Tsallis entropy [10, [15] under appropriate constraints.

On the other hand, we have also discovered the nonextensive-statisticalmechanical element in the Internet time series of the packet round-trip time measured by performing the Ping experiment [16]-[19]. We have found that statistics of the sparseness time [16] obeys the $q$-exponential distribution. The sparseness time is the quantity analogous to the time interval between earthquakes. Thus, seismicity and the Internet exhibit a similar dynamical behavior. In addition, we have also found that the Omori and Gutenberg-Richter laws also hold for "Internetquakes" corresponding to the heavily congested states of the Internet [17]-19]. This striking similarity suggests the existence of a deep root common in seismicity and the Internet. In this respect, the scale-free structure of topology 
of the Internet [20] is of central interest, and accordingly we are naturally led to examining a possible network structure underlying earthquake phenomenon.

Here, we propose the definition of the earthquake network and analyze its topological properties. Using the seismic data taken in southern California, we calculate the distribution of connectivities, the degree of separation (i.e., the characteristic path length), and the clustering coefficient of the earthquake network. We shall show that the distribution of connectivities decays as a power law, indicating that the earthquake network is scale free [21]-[24]. The degree of separation is found to take a small value between 2 and 3 , exhibiting the small-world structure of the network [25]. We shall also show that the clustering coefficient is about 10 times larger than that in the case of the completely random network, implying the complex hierarchical structure [22], [23], 25].

The present paper is organized as follows. In Sect. 2, we explain how to construct the earthquake network from the seismic data. In Sect. 3, we discuss the scale-free nature of the earthquake network constructed from the data taken in southern California. An interpretation is given to the emergence of the scale-free nature in connection with the geological feature of aftershocks. In Sect. 4, we discuss the small-world and hierarchical properties. Sect. 5 is devoted to conclusion.

\section{Earthquake Network}

Our proposal for constructing the earthquake network is as follows. A geographical area under consideration is divided into a lot of small cubic cells. A cell is regarded as a vertex when earthquakes with any values of magnitude occurred therein. Two successive events define an edge between two vertices. In this way, the complex fault-fault interaction is replaced by this edge. If two successive earthquakes occur in the same cell, they form a loop. This procedure allows us to map the seismic data to an evolving network. This construction contains a unique parameter, which is the cell size. Since there are no a priori operational rules to determine the cell size, it is essential to examine the dependencies of the network properties on this parameter. Once the cell size is fixed, the earthquake network is unambiguously defined by the seismic data [26], [27]. The earthquake network contains a few special vertices associated with mainshocks. Careful analysis of the seismic data shows that aftershocks associated with a mainshock tend to return to the locus of the mainshock geographically and consequently a stronger mainshock tends to have the larger values of connectivities contributed by more aftershocks. Accordingly, the concept of preferential attachment [21]-24] is realized by the existence of "hubs", the role of which is played by the mainshocks. This is schematically depicted in Fig. 1, in which the vertices, $A$ and $B$, may be identified with the cells containing the mainshocks. This observation leads to the reasoning that the earthquake network may be scale-free and possess the small-world structure. In what follows, we shall show that it is indeed the case. 


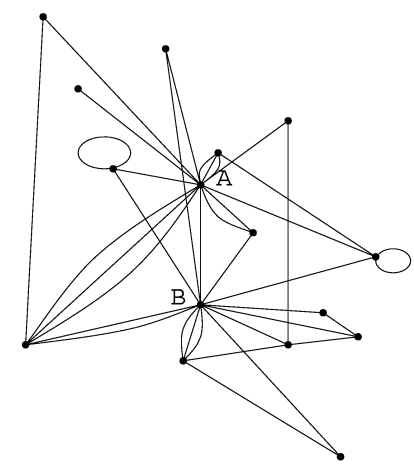

Fig. 1. A schematic description of the earthquake network. The vertices represent the cells in which earthquake occured and the edges replace the complex fault-fault interaction. $A$ and $B$ are the cells containing mainshocks and have large connectivities.

\section{Scale-Free Nature of Earthquake Network}

A key concept in the Barabási-Albert scale-free networks is the rule of preferential attachment. According to this rule, a newly created vertex is connected to the vertex $v_{i}$ with connectivity $k_{i}$ with probability

$$
\Pi\left(k_{i}\right)=\frac{k_{i}+1}{\sum_{j}\left(k_{j}+1\right)} .
$$

In [24], Albert and Barabási have discussed an exactly solvable model of an evolving random graph and have derived the analytic expression for the distribution of connectivities, $P(k)$, in the continuum limit. This solution is the Zipf-Mandelbrot-type distribution [28]

$$
P(k) \sim\left(k+k_{0}\right)^{-\gamma}
$$

with $\gamma>1$ and $k_{0}>0$, which clearly decays as a power law. In the worked examples 22, the exponent $\gamma$ ranges between 1.05 and 3.4.

It should be noted that, with identifications $q=1+1 / \gamma$ and $\kappa=(q-$ $1) / k_{0}, P(k)$ in Eq. (2) is reexpressed in terms of the $q$-exponential function as $P(k) \sim e_{q}(-k / \kappa)$, where $e_{q}(x)=(1+(1-q) x)_{+}^{1 /(1-q)}$ with the notation $(a)_{+} \equiv \max \{0, a\}$. This implies that the Albert-Barabási solution optimizes the Tsallis entropy [10], [15], $S_{q}[P]=(1-q)^{-1}\left(\int d k P^{q}(k)-1\right)$, under the appropriate constraints on the normalization of $P(k)$ and the average number of edges in the continuum limit. Relevance of Tsallis nonextensive statistical mechanics [10]-15] to a complex networks has recently been a noticed also in [29].

We have constructed the earthquake networks in the area of southern California by introducing two cell sizes, $10 \mathrm{~km} \times 10 \mathrm{~km} \times 10 \mathrm{~km}$ and $5 \mathrm{~km} \times 5 \mathrm{~km} \times 5 \mathrm{~km}$. (We have examine these two cases, since as already mentioned there are no 

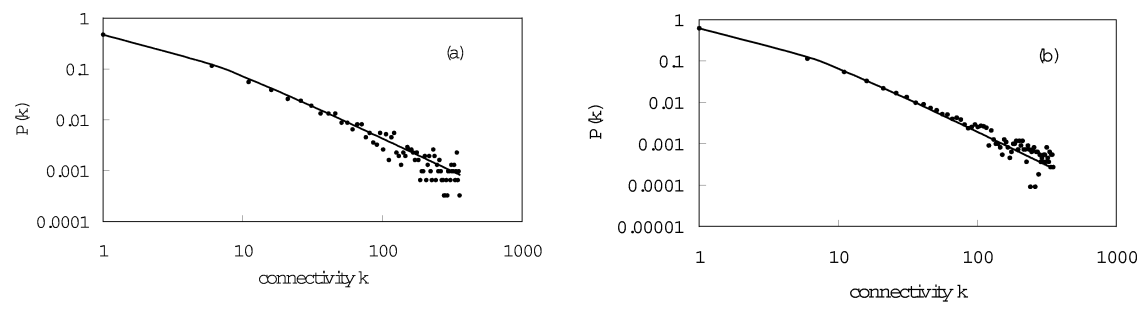

Fig. 2. The log-log plots of the distributions of connectivities. All quantities are dimensionless. The dots represent the observed data and the solid lines are drawn by using Eq. (2). (a) The cell size $10 \mathrm{~km} \times 10 \mathrm{~km} \times 10 \mathrm{~km}, \gamma=1.33( \pm 0.03)$, and $k_{0}=1.70( \pm 0.02)$. (b) The cell size $5 \mathrm{~km} \times 5 \mathrm{~km} \times 5 \mathrm{~km}, \gamma=1.61( \pm 0.03)$, and $k_{0}=2.04( \pm 0.02)$.

a priori operational rules to fix the cell size.) We have analyzed the earthquake catalog made available by the Southern California Earthquake Data Center (http://www.scecdc.scec.org/catalogs.html) covering the region $29^{\circ} 15.25^{\prime} \mathrm{N}-$ $38^{\circ} 49.02^{\prime} \mathrm{N}$ latitude and $113^{\circ} 09.00^{\prime} \mathrm{W}-122^{\circ} 23.55^{\prime} \mathrm{W}$ longitude with the maximal depth (of the foci of the observed earthquakes) $57.88 \mathrm{~km}$ in the period between 00:25:8.58 on January 1, 1984 and 15:23:54.73 on December 31, 2002. (We have taken this period since the data in 1983 are partially missing for a few months.) In Fig. 2, we present the plots of the distributions of connectivities. One appreciates that the data is well fitted by Eq.(2), and therefore the earthquake networks possess the scale-free nature. This may be interpreted as follows. The Gutenberg-Richter law, on the one hand, tells us that frequency of earthquakes with large values of moment decays as a power law. On the other hand, as already mentioned, aftershocks associated with a mainshock tend to be connected to the vertex of the mainshock, realizing preferential attachment. These imply that the scale-free nature of the distribution of connectivities may have its origin in the Gutenberg-Richter law.

We would also like to report the following further two findings regarding time evolution of the earthquake network. (i) As shown in Fig. 3, the factor, $k_{0}$, appearing in Eq. (2) is found to change in time, in contrast to the Albert-Barabási solution given in [24]. Monotonic increase of the value of $k_{0}$ is clearly observed there. (ii) The value of the exponent, $\gamma$, is ascertained to remain constant in time according to evolution of the earthquake network.

Concerning (i) above, we notice the following two points regarding consistency of time evolution with the principle of maximum Tsallis entropy. Firstly, the time-dependent problems have repeatedly been investigated within the maximum entropy principle in the literature. It is known that the maximum entropy principle is not limited to characterizing the equilibrium states in thermodynamics but can be used in much wider contexts of information theory and statistics. Secondly, as can be seen in Fig. 3, the rate of change of $k_{0}$ in the present case is less than 0.1 per a year, and therefore its time scale is much longer than that of microscopic evolution (typically about 20000 events per a year).

Closing this section, we stress the following point. We have also analyzed the data taken in Japan and have found same trends in the earthquake network [26]. 


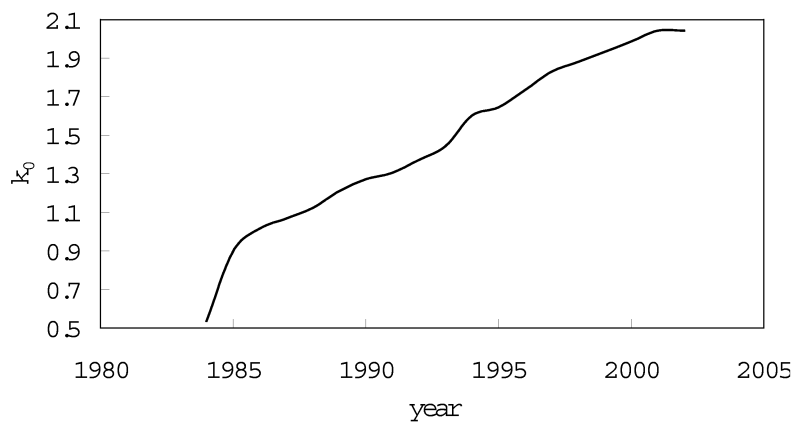

Fig. 3. Change of the value of $k_{0}$ according to evolution of the earthquake network. Here, the cell size employed is $5 \mathrm{~km} \times 5 \mathrm{~km} \times 5 \mathrm{~km}$. The value of the exponent remains constant, $\gamma=1.61$.

\section{Small-World Property of Earthquake Network}

The scale-free nature of the earthquake network implies that the network possesses the small-world and hierarchical structures. We discuss these properties in this section.

In Fig. 4, we present the degrees of separation, i.e., the characteristic path length, between an arbitrary pair of two vertices. Here, we examine the variation of the cell size from $5 \mathrm{~km} \times 5 \mathrm{~km} \times 5 \mathrm{~km}$ to $10 \mathrm{~km} \times 10 \mathrm{~km} \times 10 \mathrm{~km}$ by every $1 \mathrm{~km}$. The values of the degree of separation have been calculated by random sampling of 60 pairs of vertices. The degrees tend to slightly decrease with respect to the cell size, as it should do. The values are typically in-between 2 and 3, very small, showing the small-world nature of the earthquake network.

Next we study the clustering coefficient, $C$, proposed by Watts and Strogatz [25]. The scale-free nature of the distribution of connectivities implies that the value of $C$ is much larger than that in the case of the completely random network whose distribution of connectivities is Poissonian [21, 22, [30]. To evaluate $C$, it is essential to notice that loops attached to a single vertex should be removed and multiple edges between two vertices have to be identified with a single edge. For example, consider three vertices, $v_{1}, v_{2}$, and $v_{3}$. Suppose that they are originally connected as $v_{1} \rightarrow v_{2} \rightarrow v_{1} \rightarrow v_{2} \rightarrow v_{2} \rightarrow v_{3}$. This should be identified with $v_{1} \rightarrow v_{2} \rightarrow v_{3}$ now.

The clustering coefficient is defined as follows. Let the $v_{i}$ be the vertex which has $k_{i}-1$ neighboring vertices. At most, $k_{i}\left(k_{i}-1\right) / 2$ edges can exist between them. Calculate $c_{i} \equiv$ (number of edges of $v_{i}$ and its neighbors)/[ $\left.k_{i}\left(k_{i}-1\right) / 2\right]$. Then, the clustering coefficient is given by

$$
C=\frac{1}{N} \sum_{i=1}^{N} c_{i},
$$

where $N$ denotes the total number of vertices. In the case of the completely random network, this quantity is known to be expressed as follows [22], [23], 25]: 


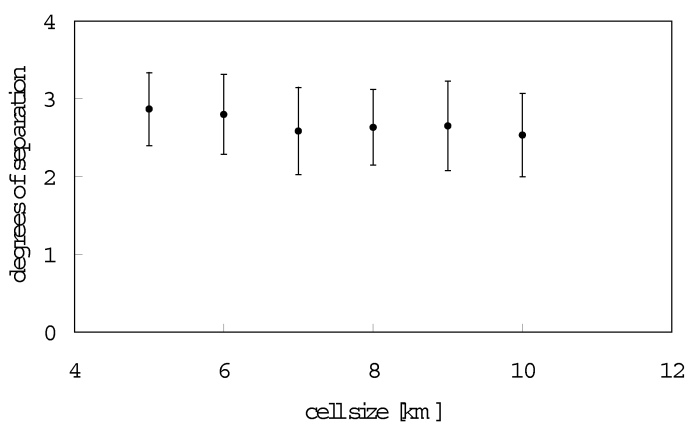

Fig. 4. The degrees of separation for various cell sizes. The values are $2.87( \pm 0.47)$ for $5 \mathrm{~km} \times 5 \mathrm{~km} \times 5 \mathrm{~km}(N=3434), 2.80( \pm 0.51)$ for $6 \mathrm{~km} \times 6 \mathrm{~km} \times 6 \mathrm{~km}(N=2731)$, $2.58( \pm 0.56)$ for $7 \mathrm{~km} \times 7 \mathrm{~km} \times 7 \mathrm{~km}(N=2165), 2.63( \pm 0.49)$ for $8 \mathrm{~km} \times 8 \mathrm{~km} \times$ $8 \mathrm{~km}(N=1827), 2.65( \pm 0.58)$ for $9 \mathrm{~km} \times 9 \mathrm{~km} \times 9 \mathrm{~km}(N=1582)$, and $2.53( \pm 0.54)$ for $10 \mathrm{~km} \times 10 \mathrm{~km} \times 10 \mathrm{~km}(N=1344)$, where $N$ denotes the number of the vertices. The error bounds are given by the standard deviations for 60 pairs of vertices chosen at random.

$$
C=C_{\text {random }}=\frac{<k>}{N} \ll 1,
$$

where $\langle k\rangle$ is the average connectivity. The point is that a complex network has the clustering coefficient which is much larger than $C_{\text {random } 25}$.

We have analyzed two subintervals of the seismic data in southern California mentioned before: (I) between 01:50:15.81 on January 30, 1992 and 05:48:10.93 on February 2, 1992, with 63 events, and (II) between 11:57:34.10 on June 28, 1992 and 20:48:22.61 on June 28, 1992, with 176 events. Seismicity in the period (I) is moderate, in contrast to the active period (II). The initial time of the period (II) is adjusted to be the event of the mainshock with M7.3 (34 $12.01^{\prime} \mathrm{N}$ latitude, $116^{\circ} 26.20^{\prime} \mathrm{W}$ longitude, and $0.97 \mathrm{~km}$ in depth), followed by a lot of aftershocks. This is why the period (II) is much shorter than (I). The cell size is taken to be $10 \mathrm{~km} \times 10 \mathrm{~km} \times 10 \mathrm{~km}$ for (I) and (II). Both of the corresponding earthquake networks have 50 vertices. The results are: $(\mathrm{I}) C_{\text {actual }}=0.680\left(C_{\text {random }}=0.046\right)$, (II) $C_{\text {actual }}=0.653\left(C_{\text {random }}=0.093\right)$. Therefore, we conclude that compared to the completely random network, the clustering coefficient is about 10 times larger, highlighting the hierarchical property of the earthquake network.

Finally, we mention that employing the method of random sampling is due to the limitation of our computational power for combinatorial problems. However, through extensive tests, we confidently believe that the results reported here are correct ones. 


\section{Conclusion}

We have constructed the complex evolving network for earthquakes in the area of southern California and have studied its topological properties. We have found that the earthquake networks have the scale-free nature in their distributions of connectivities and, thus, have presented a novel feature of seismicity as a complex critical phenomenon. We have presented an interpretation of this scalefree nature in conformity with the Gutenberg-Richter law. Then, we have studied the small-world and hierarchical properties of the earthquake network. We have shown that the degrees of separation between two vertices as the vertices take a small value between 2 and 3 . We have also calculated the clustering coefficient and have found that its actual value is about 10 times larger than that in the case of the completely random network.

We believe that these new discoveries may play an important role in modeling earthquake phenomenon [31], 32] and shed light on an aspect of seismology from a novel viewpoints.

\section{References}

1. Bak, P., Tang, C.: Earthquakes as a Self-Organized Critical Phenomenon. J. Geophys. Res. 94 (1989) 15635-15637

2. Olami, Z., Feder, H.J.S., Christensen, K.: Self-Organized Criticality in a Continuous, Nonconservative Cellular Automaton Modeling Earthquakes. Phys. Rev. Lett. 68 (1992) 1244-1247

3. Huang, Y., Johansen, A., Lee, M.W., Saleur, H., Sornette, D.: Artifactual Log Periodicity in Finite Size Data: Relevance for Earthquake Aftershocks.: J. Geophys. Res. 105 (B11) (2000) 25451-25471

4. Bak, P., Christensen, K., Danon, L., Scanlon, T.: Unified Scaling Law for Earthquakes. Phys. Rev. Lett. 88 (2002) 178501

5. Lise, S., Paczuski, M.: Nonconservative Earthquake Model of Self-Organized Criticality on a Random Graph. Phys. Rev. Lett. 88 (2002) 228301

6. Omori, F.: On the Aftershocks of Earthquakes. J. Coll. Sci. Imp. Univ. Tokyo 7 (1894) 111-216

7. Gutenberg, B., Richter, C.F.: Frequency of Earthquakes in California. Bull. Seism. Soc. Am. 34 (1944) 185-188

8. Abe, S., Suzuki, N.: Law for the Distance between Successive Earthquakes. J. Geophys. Res. 108 (B2) (2003) 2113

9. Abe, S., Suzuki, N.: Zipf-Mandelbrot Law for Time Intervals of Earthquakes. eprint cond-mat/0208344

10. Tsallis, C.: Possible Generalization of Boltzmann-Gibbs Statistics. J. Stat. Phys. 52 (1988) 479-487

11. Tsallis, C., Mendes, R.S., Plastino, A.R.: The Role of Constraints within Generalized Nonextensive Statistics. Physica A 261 (1998) 534-554

12. Abe, S., Okamoto, Y. (eds.): Nonextensive Statistical Mechanics and Its Applications. Springer-Verlag, Heidelberg (2001)

13. Kaniadakis, G., Lissia, M., Rapisarda, A. (eds.): Special Issue of Physica A 305 (2002) 
14. Gell-Mann, M., Tsallis, C. (eds.): Nonextensive Entropy-Interdisciplinary Applications. Oxford University Press, Oxford (2003)

15. Abe, S.: Stability of Tsallis Entropy and Instabilities of Rényi and Normalized Tsallis Entropies: A Basis for $q$-Exponential Distributions. Phys. Rev. E 66 (2002) 046134

16. Abe, S., Suzuki, N.: Itineration of the Internet over Nonequilibrium Stationary States in Tsallis Statistics. Phys. Rev. E 67 (2003) 016106

17. Abe, S., Suzuki, N.: Omori's Law in the Internet Traffic. Europhys. Lett. 61 (2003) $852-855$

18. Abe, S., Suzuki, N.: Gutenberg-Richter Law for Internetquakes. Physica A 319 (2003) $552-556$

19. Abe, S., Suzuki, N.: Statistical Similarity between Internetquakes and Earthquakes. Physica D (to appear)

20. Faloutsos, M., Faloutsos, P., Faloutsos, C.: On Power-Law Relationships of the Internet Topology. ACM SIGCOMM Comput. Commun. Rev. 29 (1999) 251-262

21. Barabási, A.-L., Albert, R.: Emergence of Scaling in Random Networks. Science 286 (1999) 509-512

22. Albert, R., Barabási, A.-L.: Statistical Mechanics of Complex Network. Rev. Mod. Phys. 74 (2002) 47-97

23. Dorogovtsev, S.N., Mendes, J.F.F.: Evolution of Networks. Oxford University Press, Oxford (2003)

24. Albert, R., Barabási, A.-L.: Topology of Evolving Networks: Local Events and Universality. Phys. Rev. Lett. 85 (2000) 5234-5237

25. Watts, D.J., Strogatz, S.H.: Collective Dynamics of 'Small-World' Networks. Nature (London) 393 (1998) 440-442

26. Abe, S., Suzuki, N.: Scale-Free Network of Earthquakes. Europhys. Lett. 65 (2004) $581-586$

27. Abe, S., Suzuki, N.: Small-World Structure of Earthquake Network. e-print condmat/0308208, Physica A (to appear)

28. Mandelbrot, B.B.: The Fractal Geometry of Nature. Freeman, San Francisco (1983)

29. Tadic, B., Thurner, S.: Information Super-Diffusion on Structured Networks. eprint cond-mat/0307670

30. Bollobás, B.: Random Graphs, 2nd edition. Cambridge University Press, Cambridge (2001)

31. Baiesi, M., Paczuski, M.: Scale Free Networks of Earthquakes and Aftershocks. e-print cond-mat/0309485

32. Peixoto, T.P., Prado, C.P.C.: Distribution of Epicenters in Olami-FederChristensen Model. e-print cond-mat/0310366, Phys. Rev. E (to appear) 